Check for updates

Cite this: RSC Adv., 2018, 8, 40856

Received 14th October 2018

Accepted 2nd December 2018

DOI: $10.1039 / c 8 r a 08491 b$

rsc.li/rsc-advances

\section{A NIR light-triggered pyroelectric-dominated generator based on a liquid crystal elastomer composite actuator for photoelectric conversion and self-powered sensing $\dagger$}

\begin{abstract}
Wanyuan Wei, $\$$ Jingjing Gao, $\$$ Jingfeng Yang, Jie Wei (D) and Jinbao Guo (D) *
Transducing light into electricity in photoactive materials and composites is especially attractive for light sensing and light energy harvesting. Here, we present a near-infrared (NIR) light-triggered pyroelectricbased generator by integrating a photoresponsive composite actuator composed of a liquid crystal elastomer (LCE) and graphene-doped poly(dimethylsiloxane) (PDMS) into a polyvinylidene fluoride (PVDF) film, which can effectively convert photothermal and mechanical energy into electricity. Notably, a NIR light photothermal-triggered pyroelectric effect leads to outstanding electric output performance resulting from the large temperature fluctuation induced by the contact and separation between the LCE-based composite actuator and PVDF film upon turning on or off the NIR illumination. In addition, the photothermal pyroelectric property arising from the thermal fluctuations makes the hybrid generator highly suitable as a self-powered NIR light and temperature sensor. This light-driven LCE-based hybrid generator opens a new opportunity for developing novel power generators and active sensors.
\end{abstract}

\section{Introduction}

Energy harvesting has garnered immense interest from both industry and academia due to the growing energy demands of society and the realization of autonomous and self-powered systems..$^{1-6}$ Notably, in recent years, considerable attention has been paid to functional materials converting light energy into electric energy due to their applications in many fields, such as energy harvesting, self-powered electronics and sensors. ${ }^{7-10}$ To this end, photoelectric conversion by introducing photoresponsive materials into electronic devices may provide a new means of first changing light energy into thermal energy or mechanical energy and then further converting it into electricity. ${ }^{11-21}$ As an alternative, the integration of photosensitive materials with multiphysical polymer polyvinylidene fluoride (PVDF) or its derivatives may become a popular choice. ${ }^{16-21}$ On the one hand, PVDF is an attractive material with piezoelectric, pyroelectric, and triboelectric properties and may serve as a multifunctional carrier able to simultaneously harvest different kinds of energy. In comparison to ceramic pyroelectric

Key Laboratory of Carbon Fibers and Functional Polymers, Ministry of Education, Beijing Engineering Research Center for the Synthesis and Applications of Waterborne Polymers and College of Materials Science and Engineering, Beijing University of Chemical Technology, Beijing, 100029, China. E-mail: guojb@mail. buct.edu.cn

$\dagger$ Electronic supplementary information (ESI) available. See DOI: 10.1039/c8ra08491b

\$ The authors contributed equally to this work. materials, PVDF has mechanical flexibility, high strain and transparency, which lead to a wide range of applications, such as flexible energy conversion devices, wearable devices and other electric devices. ${ }^{22-27}$ On the other hand, among the various types of photoactuators with integrated optical-to-mechanical energy conversion capacity, including those constructed from gel, elastomer, crystal or liquid crystal (LC) materials, ${ }^{28}$ photochemical- or photothermal-driven LC elastomer (LCE) actuators exhibit excellent photoactuation performance due to their unique ability to control actuation rapidly, precisely and remotely. ${ }^{29-43}$ Thus far, two studies on the development of photoelectric energy-conversion composite systems by integrating LCE-based composite photoactuators have been reported. ${ }^{\mathbf{1 7 , 1 8}}$ However, one of the main challenges for these systems lies in their limited photoelectric conversion efficiency (voltage output at the millivolt level). To date, an LCE-based photoelectric energy-conversion system that is based mainly on the pyroelectric effect and that produces high photothermal heat-to-electricity conversion has not been explored.

In this work, we demonstrate an NIR light-triggered pyroelectric-based generator by associating a novel photoresponsive LCE composite actuator with a multifunctional PVDF film. A schematic illustration of the generator is presented in Fig. 1a. Here, pyroelectric-dominated photoelectric conversion is achieved by coupling the photomechanical property inherent to the LCE-based composite actuator with the multiphysical properties of the PVDF film. To realize energy transfer from light to electricity, we fabricate an LCE composite 
(a)
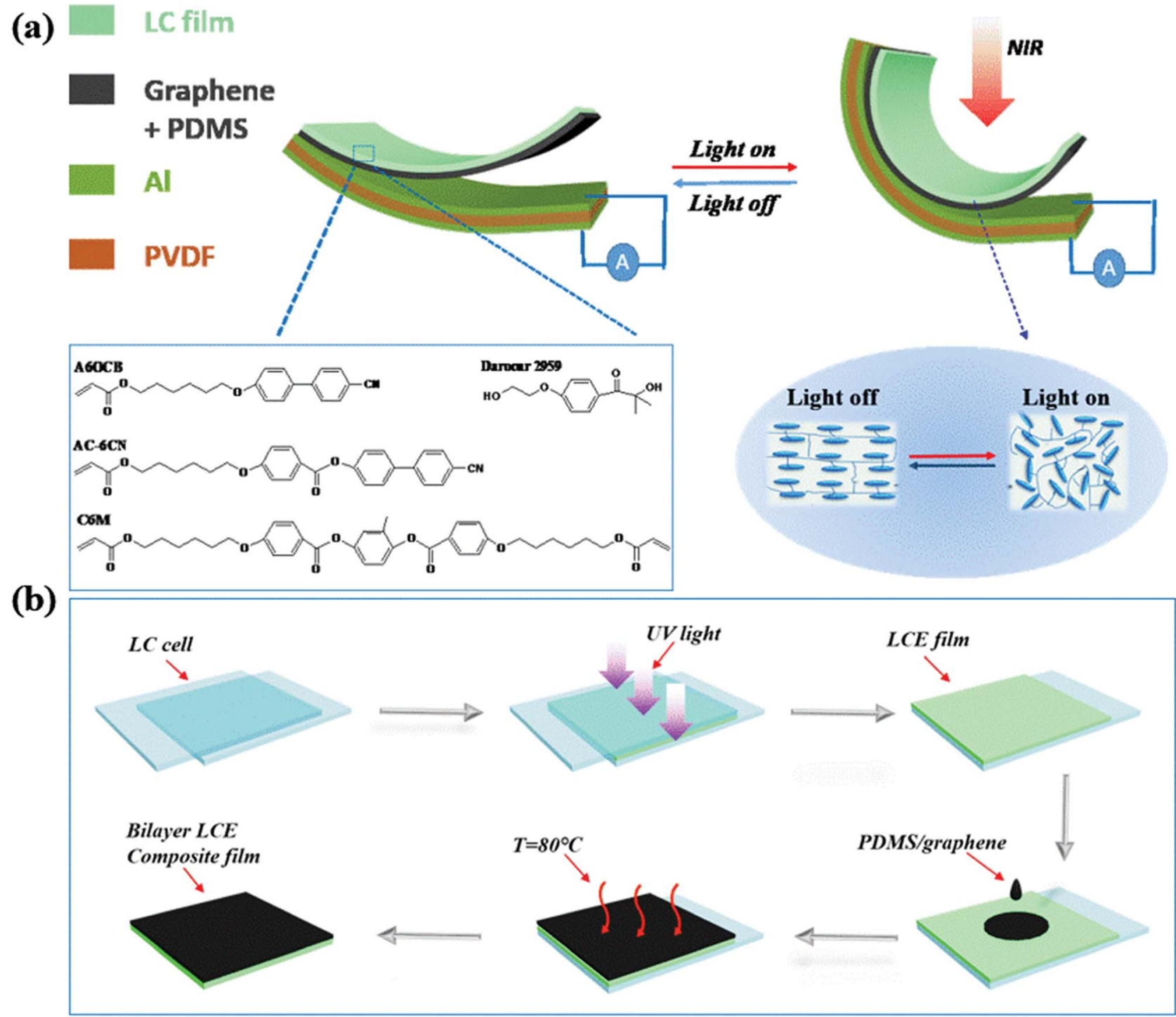

Fig. 1 (a) A schematic illustration of the hybrid generator device. The inset images, left: chemical structures of the materials used for the fabrication of the LCE; right: photograph of the cross-linked LCE before and after NIR light irradiation. (b) The schematic photogram of the fabrication process of the bilayer LCE-based composite film.

actuator by casting graphene-doped PDMS on an LCE layer, and the resultant LCE-based photoactuator with a bilayer structure exhibits fast and strong bending and unbending when subjected to and removed from $980 \mathrm{~nm}$ NIR light exposure. Furthermore, under periodic irradiation with $980 \mathrm{~nm}$ NIR light, the LCE-based actuator and PVDF film in the designed generator exhibit reversible contacting and separation, causing a large temperature fluctuation and hence producing a desirable change in polarization intensity of the PVDF film as well as different triboelectric polarities between the two sections. The investigation of the working mechanisms reveals that the large output voltage/current signals of the hybrid generator mainly stem from the pyroelectric effect. Finally, this generator constructed from photoresponsive LCE composite actuator is demonstrated as a self-powered NIR light and temperature sensor. This study is the first attempt to construct a light-driven pyroelectric-based generator using a photomechanical LCE- based composite actuator. Indeed, the results suggest a facile and effective method for developing LCE-based photoactuator materials in the capture and sensing of light.

\section{Results and discussion}

\subsection{Fabrication and deformation behavior of LCE-based} composite actuator

To fabricate a bilayer photoactuator composed of one active LCE layer and one graphene-doped PDMS composite layer (Fig. 1a), a planar-aligned LCE layer was first prepared using a UV-light-induced photopolymerization process in a planar cell as shown in Fig. 1b. A schematic of the cross-linked polymer network after UV curing is illustrated in the right inset image of Fig. 1a. Herein, the chemical structures of the components of the LCE precursor used to prepare the LCE layer are shown in the left inset image of Fig. 1a. After one side of the glass 
substrate was removed from the LC cell, the PDMS precursor doped with $5.0 \mathrm{wt} \%$ graphene sheets and trace amounts of $n$ hexane was cast onto the LCE layer. Then, it was heated at $80^{\circ} \mathrm{C}$ for $1.0 \mathrm{~h}$ to induce curing of the PDMS. Finally, a self-supported bilayer composite film was obtained by eliminating the other side of the glass substrate. All experimental details are available in the Experimental section.

Fig. 2a shows a schematic diagram of the obtained LCE/ graphene-PDMS bilayer composite film. A cross-sectional scanning electron micrograph (SEM) image of the bilayer composite film is shown in Fig. 2b. The graphene-doped PDMS layer and LCE were compactly contacted with each other, and the thickness of the LCE layer (bottom) and the graphenePDMS layer (top) were approximately 27.6 and $37.5 \mu \mathrm{m}$, respectively. The surface of the graphene-PDMS composite layer was smooth, and the graphene sheets were uniformly distributed in the PDMS matrix. We have two reasons for designing a photoactuator with such a special structure: (1) PDMS is a good candidate as a frictional layer in a graphenedoped PDMS composite layer, and graphene can efficiently absorb and transform NIR light into thermal energy, thereby serving as an effective heater to induce the phase transition of the LCE layer and to heat the PVDF layer; (2) in contrast with previous studies, this study added graphene as a heater to the PDMS matrix instead of the LCE layer because the graphenedoped PDMS could also be expected to improve the output voltage/current of the generator. ${ }^{44}$ As illustrated in Fig. $2 c$, when the NIR light is incident to the LCE layer, bending deformation of the photoactuator could be clearly observed, and the bending actuation of the bilayer film was found to be fast and reversible. In a typical bilayer composite film with a length of $2.0 \mathrm{~cm}$, the time needed to bend to the film to its maximum curvature is approximately $11.4 \mathrm{~s}$, and the recovery time is approximately 11.2 $\mathrm{s}$ after turning off the NIR light, as shown in Fig. 2c (see details in Movie 1, ESI $\dagger$ ). Moreover, when the NIR light is on, the temperature of the LCE-based bilayer film could reach $104.8^{\circ} \mathrm{C}$ (Fig. 2d), which is well beyond the $T_{\mathrm{g}}$ of the LCE layer (Fig. S1, $\left.\mathrm{ESI}^{\dagger}\right)$. The relationship between curvature and film temperature is demonstrated in Fig. 2e. It is clear that the curvature of the composite film becomes large as the temperature of the film surface increases. In our design, doped graphene is a good photothermal conversion additive in the photoresponsive bilayer actuators and is capable of absorbing NIR light and transforming it into thermal energy. It is concluded from the above results that the NIR light could be absorbed immediately by the graphene sheets present at the interface of the LCE and graphene-PDMS layers due to the good transparency of the LCE layer. Furthermore, the converted heat energy is then transported to both the LCE layer and the PDMS/graphene layer, as shown by the schematic view of the heat transfer process in Fig. 2f. This analytical result is essentially consistent with the experimental measurement shown in Fig. 2d, which is also similar to another photo-driven soft actuator. ${ }^{45}$ In the geometry studied here, graphene doped in PDMS can efficiently absorb and transform NIR light into thermal energy, thereby inducing the LCE N-I phase transition and reducing the order parameter in the LC molecules. As a result, the planar-aligned LCE layer in the bilayer film underwent significant in-plane contraction. Because the LCE layer contracted along the alignment direction while the PDMS layer remained unchanged instead of the difference in thermal expansion coefficient between the two layers, the bilayer photoactuator bent towards the LCE layer. This analysis was supported by the fact that almost no change in curvature was observed when the NIR light intensity exceeded

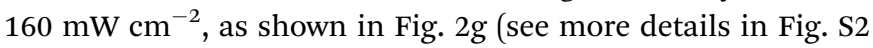
and S3, ESI $\dagger$ ). This bending mechanism is similar to that of SWNT-LCE composite/silicone bilayer and quasi-bilayer LCEbased composite films in previous reports. ${ }^{37,46,47}$ Fig. $2 \mathrm{~h}$ shows the variation in the flat and bending angles with cycling before and after exposure to NIR light. Notably, the bilayer photomechanical actuator remained intact even after over 20 cycles of reversible bending and unbending. This result suggested that a reversible deformation process could be achieved after cycling. In fact, NIR light-induced bending actuation could be controlled by adjusting the NIR light intensity, graphene content and layer thickness. In this study, the LCE-based photoactuator exhibited ideal performance through a series of comparative experiments, including the photoactuator containing $5.0 \mathrm{wt} \%$ graphene in a PDMS matrix.

\subsection{Pyroelectric-based generator constructed from LCE- based composite actuator}

Fig. 3a and $\mathrm{a}^{\prime}$ present photographs of the pyroelectricdominated generator in the initial state and the maximum bending state, respectively. In our design, the generator was composed of an LCE-graphene-doped PDMS bilayer photoactuator and a PVDF film with aluminum (Al) electrodes on both surfaces. Importantly, the Al-based metallic electrodes on both sides of the PVDF layer have high thermal and electrical conductivity, which enables efficient heat transfer at the interface between the Al electrode and the PVDF film. In this study, these two sections were bonded together on the one end, and the experimental details are available in the Experimental section. Fig. $3 \mathrm{~b}$ and $\mathrm{b}^{\prime}$ show the measured Al surface temperatures upon exposure to the NIR light in the on and off states during the bending deformation. The obtained generator clearly still exhibited bending deformation upon irradiation with $980 \mathrm{~nm}$ NIR light, and the dynamic process is also available (see details in Movie 2, ESI $\dagger$ ). When the generator underwent bending upon irradiation with NIR light, the distance between the bilayer photoactuator and Al-coated PVDF film decreased gradually until the last contact, while the opposite process occurred upon turning off the NIR light. This reversible contact and separation between the LCE-based composite photoactuator and Al-coated PVDF film could be attributed to the bending deformation of the photothermal-driven LCE-based photoactuator, as discussed above. To reveal the photoelectric conversion performance, the generator was irradiated by periodically turning on and off a $980 \mathrm{~nm}$ NIR laser with a fixed intensity of $160 \mathrm{~mW} \mathrm{~cm} \mathrm{~cm}^{-2}$ in the following experiment. The equipment used in the measurement of output signals is provided in Fig. S4. $\dagger$ As shown in Fig. 3c, a positive voltage is observed when the composite film bends accompanied by the 


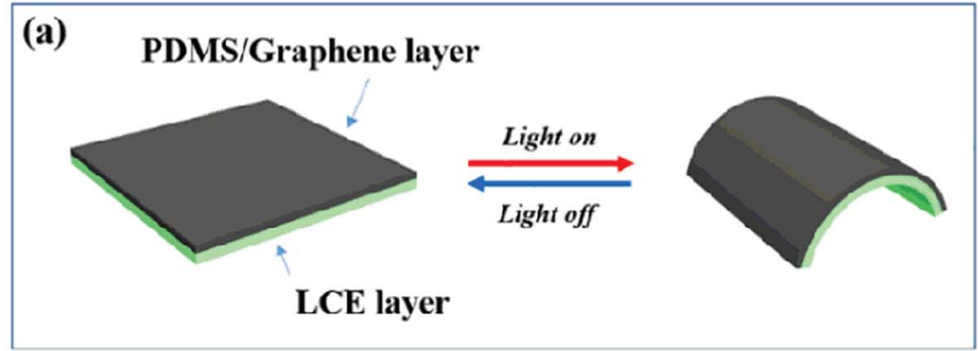

(b)

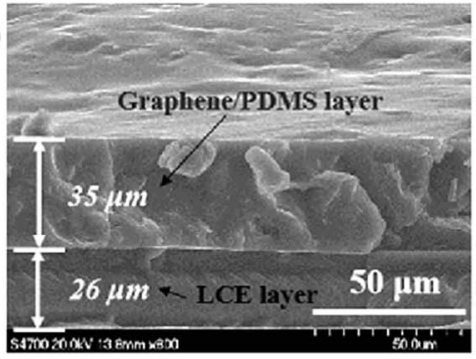

(c)
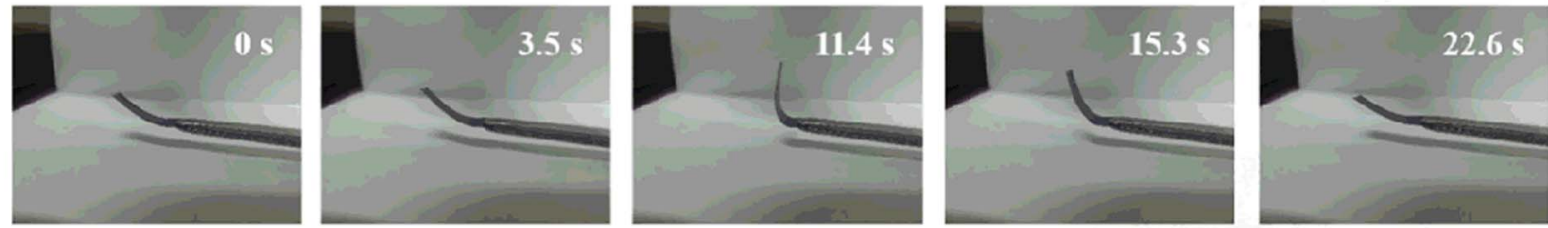

(d)
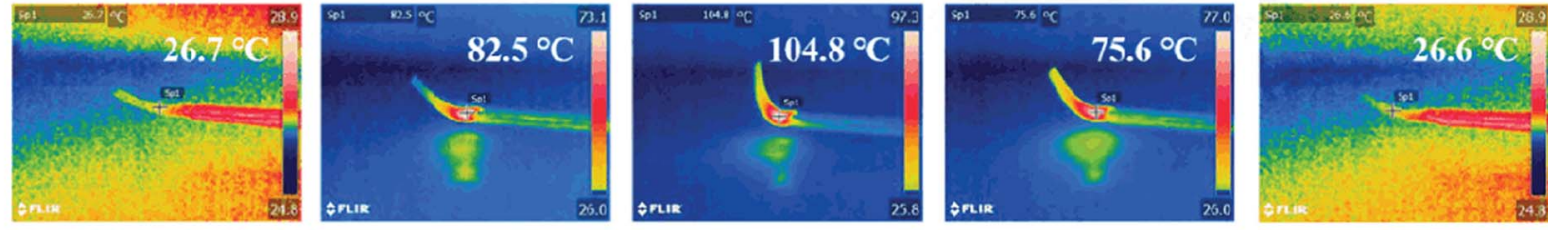

(e)

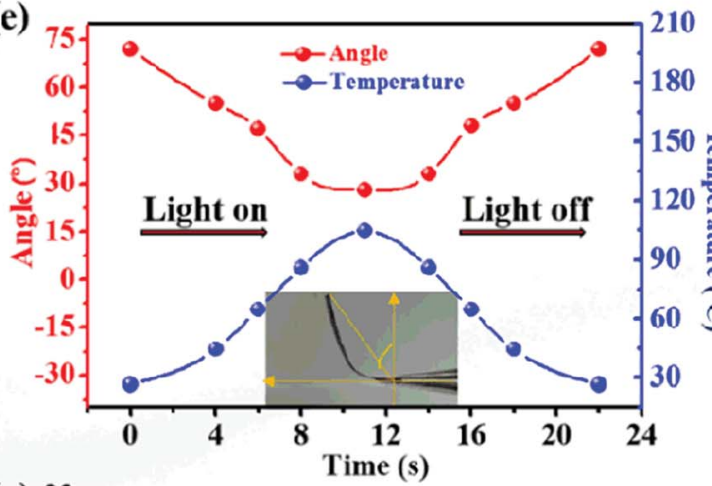

(g)

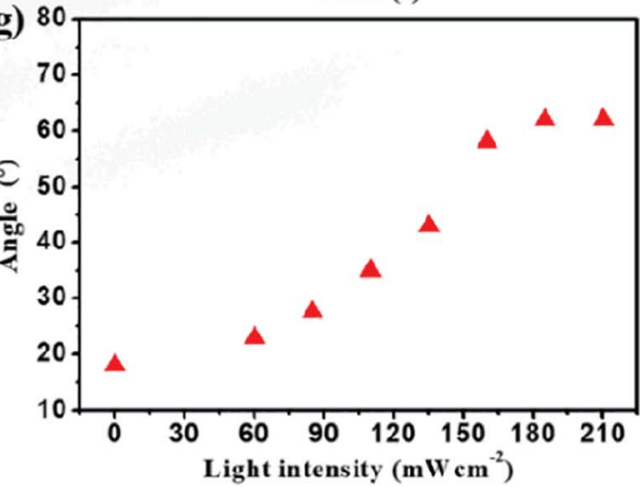

(f)

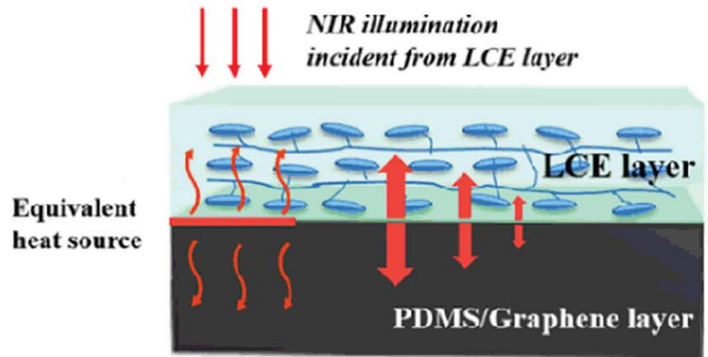

(h)

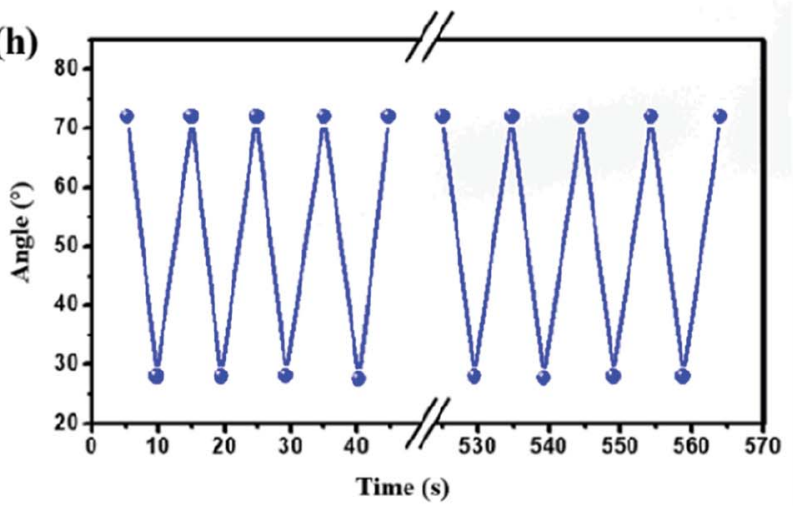

Fig. 2 (a) Schematic diagram of the obtained LCE/graphene-PDMS bilayer composite film. (b) A cross-section view of SEM image of the bilayer composite film. (c) The bending deformation process of the LCE-based photoactuator. (d) The surface temperature of the photoactuator measured by thermal image recording. (e) The variations in bending curvature and surface temperature as a function of irradiation time. (f) $A$ schematic view of the heat transfer process in the bilayer photoactuator. (g) The bending angle as a function of $980 \mathrm{~nm}$ NIR light intensity. (h) Variation in the flat and bending angles with cycling before and after exposure to NIR light.

contact between the top section and bottom section upon irradiation with $980 \mathrm{~nm}$ NIR light, while a negative voltage appears when the composite film recovers to the original state of separation between the top and bottom parts after the NIR laser light is turned off. Fig. 3c and d show the measured output voltage and output current signals of the generator, respectively. The results indicate that the output voltage is approximately $20 \mathrm{~V}$ and that the output current is approximately $6.0 \mathrm{nA}$. 
(a)

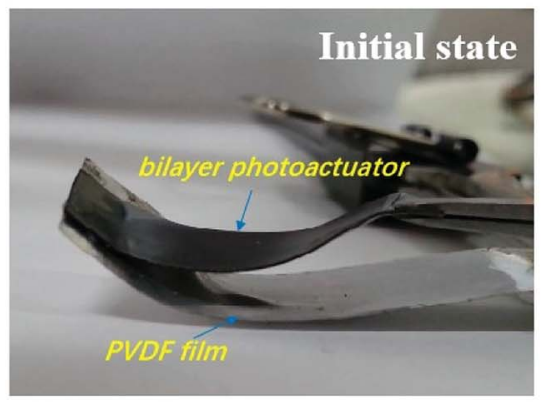

(b)

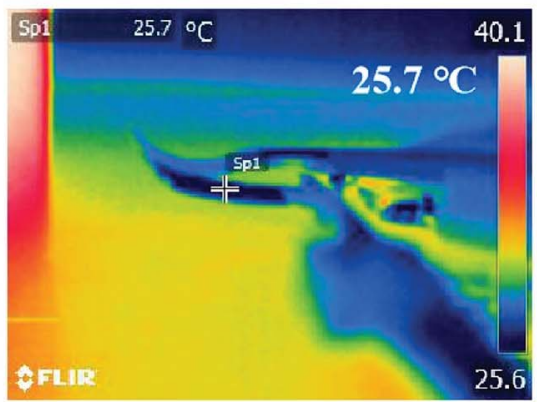

(c)

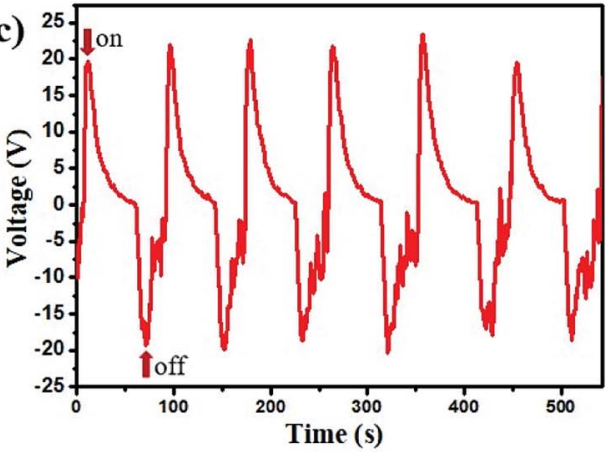

(a')

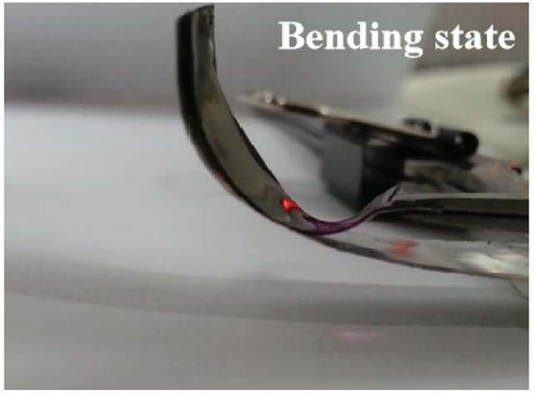

(b')
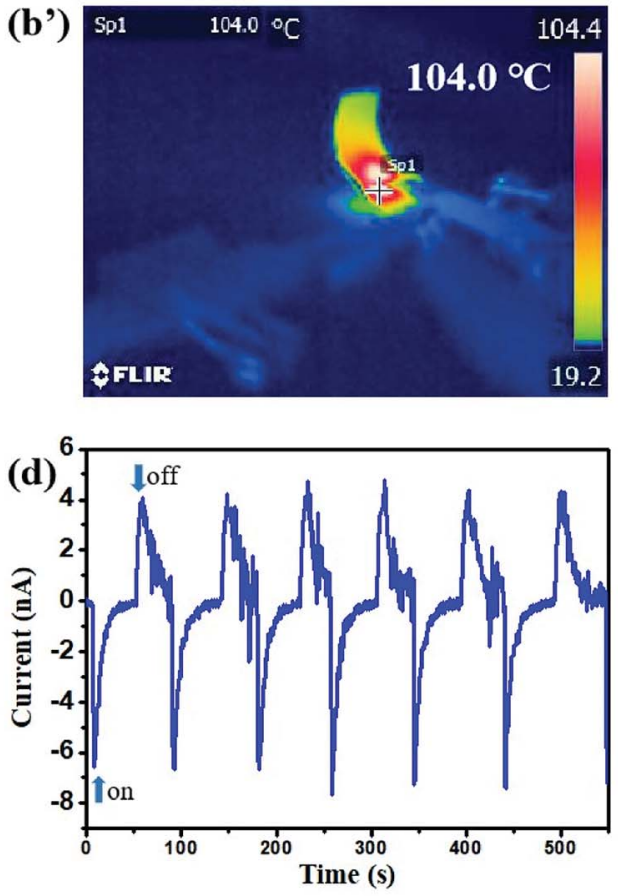

Fig. 3 (a) and ( $\left.a^{\prime}\right)$ Photo images of the hybrid generator at initial state and the maximum bending state, respectively. (b) and ( $\left.b^{\prime}\right)$ The measured surface temperatures of Al-coated PVDF film upon the NIR light on and off states during the bending deformation. (c) and (d) Output voltage and current signals of the hybrid device, respectively.

In addition, this generator can produce voltage and current signals at a certain frequency when it was bent and released reversibly together with separation and contact between the top and bottom sections. Specifically, the analysis of one bending deformation cycle is provided (Fig. S5, ESI $†$ ). The response times of both the voltage and current generation were consistent with that of the bending deformation of the generator.

On the basis of the experimental data and analysis, it is reasonable to postulate that such a large output electric signal (especially voltage output) in the device may be mainly attributed to pyroelectric effect. To verify this point, we designed an experiment to independently evaluate this effect. The LCE composite photoactuator and PVDF section was attached together without bending deformation, followed by the irradiation by periodically turning on and off a $980 \mathrm{~nm}$ NIR laser. The output voltage and current signals derived from only the pyroelectric effect are shown in Fig. 4a and b, respectively. In comparison with the generator data, the results clearly show that the pyroelectric effect is the primary contributor to the overall output signals. As shown in Fig. $3 b$ and $b^{\prime}$, a large temperature change $\left(\Delta T=78.3^{\circ} \mathrm{C}\right)$ occurred on the surface of the PVDF surface during the separation and contact process. This temperature change produces a significant pyroelectric effect and thus generates a large output voltage signal as well as a current signal. Furthermore, compared with the pure PVDF film by directly applying periodic NIR light irradiation, the pyroelectric voltage output of the generator demonstrated here increases by about 5 times and the current output raises by around 1.5 times (see more details in Fig. S6, ESI $\dagger$ ). Notably, the induced change in temperature (thermal gradient) in a pyroelectric device is highly desirable for effective conversion of thermal radiation energy to electric energy. ${ }^{24}$ In this system, the large temperature change derived from heat transfer during the separation and contact process between the photoactuator and PVDF film had a positive effect on the energy conversion. Herein, the designed structure is a unique innovation for achieving highly efficient pyroelectric conversion. It should also be noted that this work utilizes a simple PVDF film to show the pyroelectric effect, and it is not difficult to further improve the output performances of the device, such as structuring 


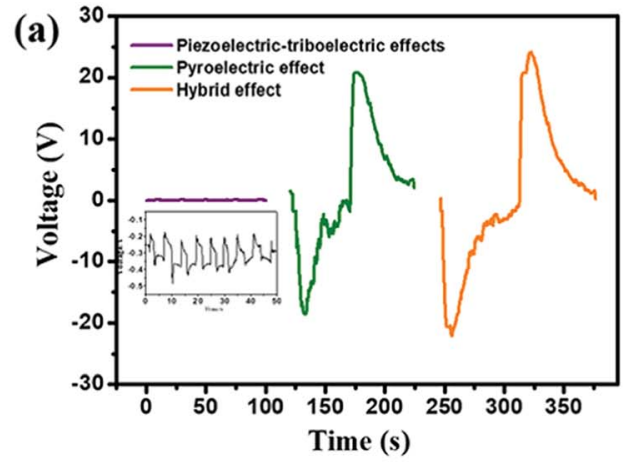

(c)
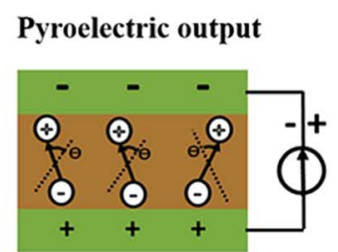

$\mathrm{dT} / \mathrm{dt}=\mathbf{0}$

(d)

Triboelectric output

(e)

\section{Piezoelectric output}

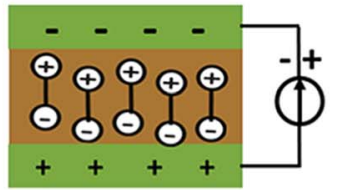

(b)

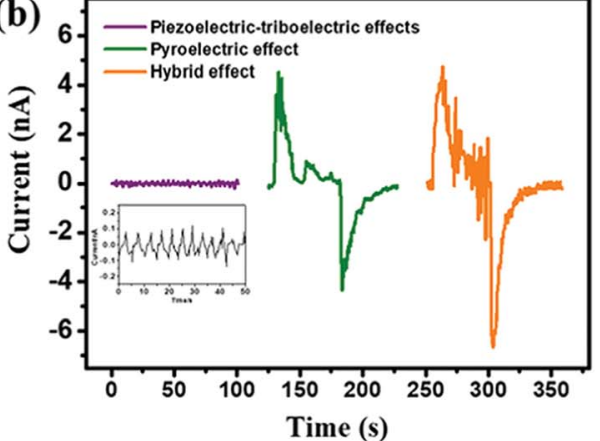

)

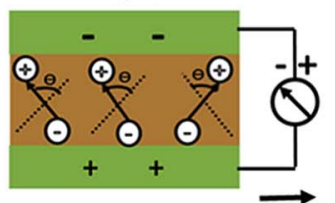

$\mathrm{dT} / \mathrm{dt}>\mathbf{0}$

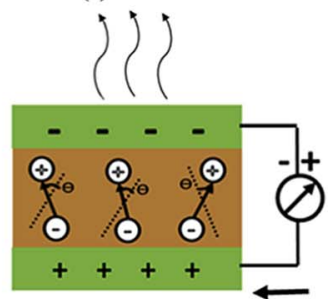

$\mathrm{dT} / \mathrm{dt}<0$

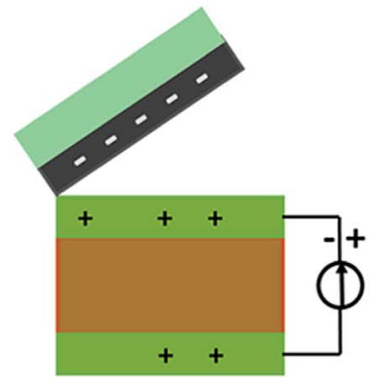

$+$

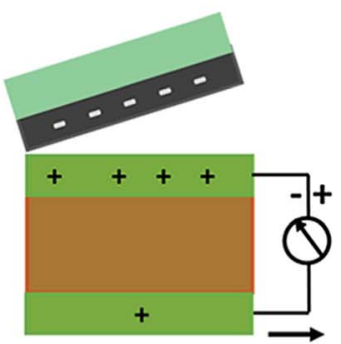

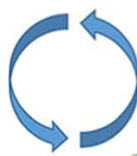
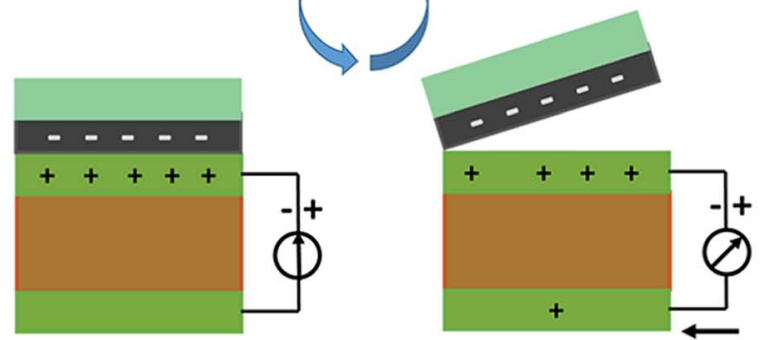

Fig. 4 (a) The output voltage and (b) current signals derived from the triboelectric-piezoelectric, pyroelectric and hybrid effects, respectively. (c)-(e): Schematic illustration of the working mechanisms of the pyroelectric, triboelectric and piezoelectric effects.

pyroelectric materials with at multiple scales. ${ }^{48}$ In addition, the bending deformation accompanying the contact and separation process may also produce output electric signals. As depicted in Fig. $4 \mathrm{a}$ and $\mathrm{b}$, the output voltage and current derived from the triboelectric-piezoelectric effects could be obtained when similar bending processes were carried out without NIR light irradiation. Although the values are much smaller than those contributed by the pyroelectric effect, it could also be confirmed that triboelectric-piezoelectric effects make a positive contribution to the output electric signals in the hybrid device (the 
inset images of Fig. 4a and b). Subsequently, the pyroelectric, triboelectric and piezoelectric output signals from the generator were integrated to produce a total electric output voltage of up to approximately $20 \mathrm{~V}$ under the concurrence of a thermal gradient (heating and cooling mode), friction (contact and separation) and deformation (bending-unbending mode).

The working mechanisms of the hybrid device was then studied in detail. Fig. 4c explains the pyroelectric output of the generator. In the device structure, the PVDF film is placed upward in the polarization direction. During the reversible bending process accompanied by the contact between the two parts upon NIR light irradiation, the heat generated from the high radiation absorption of the graphene may be transferred from the graphene-doped PDMS layer in the LCE-based photoactuators to the PVDF film, thereby resulting in a decrease in the dipole moments and a reduction in the polarization density in the PVDF film. Consequently, the potential between the top and bottom electrodes is generated in an open-circuit state, and an induced current occurs between the electrodes under shortcircuit conditions. After the LCE-based photoactuator and PVDF film separate upon turning off the NIR light, the increase in the polarization density resulting from the heat dissipation from the PVDF film induces a flow of electrons in the reverse direction. As shown in Fig. 4d, the triboelectric effect originates from the coupling of triboelectrification and electrostatic induction. Specifically, upon contact/friction between the two parts of the hybrid generator in response to NIR light irradiation, the electrons in the graphene-doped PDMS layer may be transferred to the surface of the $\mathrm{Al}$ layer because of the difference in triboelectric polarity between these two layers. Furthermore, after the separation of the graphene-doped PDMS layer and the $\mathrm{Al}$ metal layer, the formed triboelectric charges induce the flow of electrons from the top $\mathrm{Al}$ electrode to the bottom $\mathrm{Al}$ electrode due to the electrostatic induction effect. Eventually, the output current/voltages resulting from the triboelectric effect could be observed. In addition, when the bending deformation of the PVDF film happens, the reduced volume of the PVDF film leads to an enhancement in the polarization density. Thus, the current flows from the top electrode to the bottom electrode. That is, the piezoelectric effect can also produce detectable output current/voltage signals during the bending/unbending process, as depicted in Fig. 4e. It should be noted that the current signal direction of the piezoelectric effect is opposite to that of the triboelectric effect. However, the output signals derived from these two effects have the same direction as the pyroelectric effect (Fig. S7, ESI $\dagger$ ), suggesting a positive contribution to the overall signals. The analysis result is also consistent with the experimental data demonstrated above. All the above observations mean that the pyroelectric effect is dominant in electric output of the generator, while the triboelectricpiezoelectric effects could not be ignored.

\subsection{Self-powered NIR light- and temperature- sensors}

The photoelectric-converting device could be used as a selfpowered NIR light sensor for intensity monitoring. It is well known that the intensity of $980 \mathrm{~nm}$ NIR laser light is a vital indicator for NIR light sources. Here, we use the composite
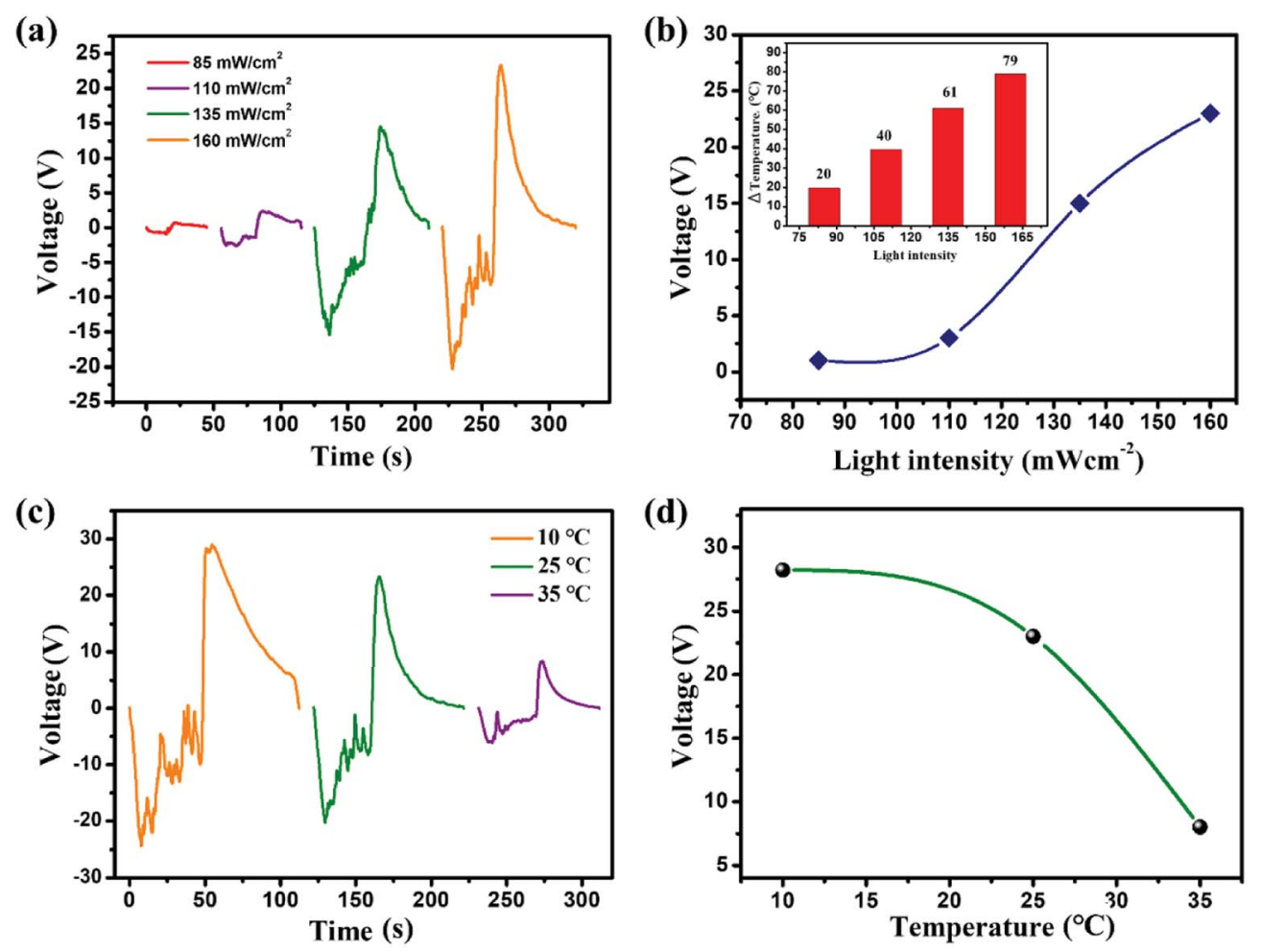

Fig. 5 (a) The output voltage of the hybrid generator under different NIR-light intensity (the ambient temperature is $25^{\circ} \mathrm{C}$ ). (b) The output voltage varies with $980 \mathrm{~nm}$ NIR light intensity with a fitting line. The inset image: the temperature changes of the PVDF film at different light intensity. (c) The output voltage of the hybrid generator at different temperature. (d) The output voltage varies with the ambient temperature with a fitting line. 
device to detect four intensities of $980 \mathrm{~nm}$ NIR light: $85 \mathrm{~mW}$ $\mathrm{cm}^{-2}, 110 \mathrm{~mW} \mathrm{~cm}{ }^{-2}, 135 \mathrm{~mW} \mathrm{~cm}^{-2}$ and $160 \mathrm{~mW} \mathrm{~cm}^{-2}$. Fig. $5 \mathrm{a}$ shows the open-circuit voltages of the device in four states at an ambient temperature of $25{ }^{\circ} \mathrm{C}$. It is clearly shown that NIR light intensity can be directly reflected by the output voltage signals. Moreover, the intensity of the output electrical signals basically presented an increasing trend as the NIR light intensity increased, as shown in Fig. 5b. This phenomenon should be ascribed to the larger temperature difference induced by a higher NIR light intensity, as shown in the inset image in Fig. 4b. From the above experimental results, it may be concluded that the output electrical signals of the photoelectric device can directly record the intensity of the NIR light. In addition, this photo-conversion device could also be promising for environmental temperature detection because the output electrical signals of the device are closely related to the temperature. Under a certain NIR light intensity $(160 \mathrm{~mW}$ $\mathrm{cm}^{-2}$ ), the output level of the device as a function of ambient temperature was investigated, and the result is shown in Fig. 5c and $d$. The results indicate that the open-circuit voltage of the device increases as the environment temperature decreases. The reason for this behavior is that the larger temperature difference at low temperature can increase the pyroelectric conversion effect. These performances suggested that the device could also serve as a self-powered sensor for environmental temperature monitoring. Additionally, it was shown that the device could be employed as a generator to drive another electric device, as presented in Fig. S8 and S9. $\dagger$ Finally, on the basis of the time dependence of the electric signals during the bending deformation process, the device could be employed to measure the movement rates of an object, as shown in Fig. S10 and S11. $\dagger$ These demonstrations further show the potential of the photoelectric conversion device for practical applications.

\section{Conclusions}

In summary, we have developed a NIR light-triggered generator composed of a composite photoactuator comprising an LCE/ graphene-doped PDMS bilayer structure and an Al-coated PVDF film. The generator is able to work not only as an efficient photoelectric energy converter but also as a self-powered sensor. The photoinduced contact and separation between the LCEbased photoactuator and the PVDF film in the hybrid generator efficiently converts photothermal and mechanical energy into electricity. In the case of a generator in which the length and width of the photoactuator are $2.0 \mathrm{~cm}$ and $0.6 \mathrm{~cm}$, respectively, it exhibits a high output voltage in excess of $20 \mathrm{~V}$ and a reasonable output current of $6.0 \mathrm{nA}$. In particular, the large electric output signals of the generator were mainly attributed to the considerable pyroelectric energy transformation, which was caused by the large temperature fluctuations arising from heating and cooling during the contact and separation between the two sections of the hybrid generator. Furthermore, the generator was also demonstrated as a multifunctional selfpowered sensor for detecting both NIR light intensity and ambient temperature variation. Thus, the proposed work provides a feasible way to develop new light-driven photoelectric conversion devices displaying good performance as both power generators and active sensors.

\section{Experimental section}

\subsection{Materials}

Most of the chemicals and solvents were obtained from commercial sources. The monoacrylate LC monomers AC-6CN and A6OCB were purchased from Bayi Space LCD. The diacrylate LC monomer C6M was synthesized in our lab according to the previous report. ${ }^{49}$ And ${ }^{1} \mathrm{H}-\mathrm{NMR}$ of $\mathrm{C} 6 \mathrm{M}$ is shown in Fig. S12. $\dagger$ The photoinitiator Darocur 2959 was purchased from J\&K Scientific Ltd. Graphene was purchased from Hefei Vigon Material Technology Co., Ltd. PDMS solution (Sylgard 184, Dow Corning) contained both the elastomer and the curing agent at a weight ratio of $10: 1$. Poly(vinylidene fluoride) (PVDF) coated with aluminum layers on both sides was purchased from Goodfellow Cambridge Limited.

\subsection{Preparation of the LCE film}

The LCE precursor was prepared by dissolving the LC monomers and photoinitiator in dichloromethane in the following ratios: $30 \mathrm{wt} \% \mathrm{AC}-6 \mathrm{CN}, 30 \mathrm{wt} \% \mathrm{A6OCB}, 40 \mathrm{wt} \% \mathrm{C} 6 \mathrm{M}$ and $2.0 \mathrm{wt} \%$ Darocur 2959. A blank LC cell was made by gluing two glass substrates coated with isopropanol-treated polyvinyl alcohol (PVA) alignment layer as top and bottom substrate, respectively. Subsequently, the cell was filled with LCE precursor in the isotropic phase $\left(120^{\circ} \mathrm{C}\right)$ and cooled down to the nematic phase $\left(95{ }^{\circ} \mathrm{C}\right)$. Finally, the LCE precursor was polymerized for $5.0 \mathrm{~min}$ by UV light irradiation with an illumination intensity of $5.0 \mathrm{~mW} \mathrm{~cm} \mathrm{~cm}^{-2}$.

\subsection{Fabrication of the LCE composite actuator}

First, $5.0 \mathrm{wt} \%$ of the graphene sheets were added to PDMS precursor and mixed by a mechanical blender for $3.0 \mathrm{~h}$. Next, the curing agent was added to the mixture under stirring, and then the mixture was spin-coated on the LCE film at a rotation speed of $2000 \mathrm{rpm}$, followed by curing at $80^{\circ} \mathrm{C}$ for $2.0 \mathrm{~h}$. Finally, the LCE-based composite actuator was peeled from the substrate. In a typical case, the length and width of the LCEbased composite actuator are approximately $20 \mathrm{~mm}$ and 6 $\mathrm{mm}$, respectively.

\subsection{Fabrication of the pyroelectric-based generator}

The photoactuator and Al-coated PVDF film were bonded together on one end using PVA glue. Meanwhile, the other ends of the photoactuator and Al-coated PVDF film were fixed by the tweezers, leaving a gap between them. Hence, reversible periodic contact and separation between the photoactuator and Alcoated PVDF film occurred upon NIR light irradiation. In addition, the Al electrode on both sides of the PVDF film was bridged with a fine wire conductor for connection with the external circuit. The size of the PVDF film is approximately $28 \mathrm{~mm} \times 10 \mathrm{~mm}$ (length and width). 


\subsection{Measurements}

The mesomorphic properties of the LC monomer mixture and the LCE-based film were examined by a polarizing optical microscope (POM, Leica, DM2500P) with a hot stage calibrated with an accuracy of $\pm 0.1{ }^{\circ} \mathrm{C}$ (Linkam, THMS-600). The morphologies of the LCE composite film were observed by SEM (Hitachi S-4700). The temperature changes in the LCE-based photoactuator and hybrid generator were measured by a thermal imaging camera (FLIR E50). The output voltage and current signals of the hybrid generator were tested by a source meter (Keithley 2450) and an electrochemical workstation (CHI $660 \mathrm{E})$.

\section{Conflicts of interest}

There are no conflicts to declare.

\section{Acknowledgements}

This work was supported by the National Natural Science Foundation of China (Grant no. 51773009 and 51373013). The authors thank Dr Xiaokang Ding from Beijing Advanced Innovation Center for Soft Matter Science and Engineering of Beijing University of Chemical Technology for his help and support in the measurement of the surface temperatures of the photoactuator and hybrid generator measured by a thermal imaging camera.

\section{References}

1 P. Cheng, G. Li, X. W. Zhan and Y. Yang, Nat. Photonics, 2018, $12,131$.

2 G. Hodes, Science, 2013, 342, 317.

3 S. Zhang, X. Yang, Y. Numata and L. Han, Energy Environ. Sci., 2013, 6, 1443.

4 R. L. Huang, M. L. Huang, X. F. Li, F. An, N. Koratkar and Z.-Z. Yu, Adv. Mater., 2018, 30, 1707025.

5 Z. L. Wang, G. Zhu, Y. Yang, S. H. Wang and C. F. Pan, Mater. Today, 2012, 15, 532.

6 F. R. Fan, W. Tang and Z. L. Wang, Adv. Mater., 2016, 28, 4283.

7 M. heldon, V. Jorik, A. Brown, P. Albert and H. Atwater, Science, 2014, 346, 828.

8 D. Francis, A. Amin, M. El-Khouly, N. Subbaiyan, M. Zandler and F. Shunichi, J. Am. Chem. Soc., 2012, 134, 654.

9 C. Zhang, Z. H. Zhang, X. Yang, T. Zhou, C. B. Han and Z. L. Wang, Adv. Funct. Mater., 2016, 26, 2554.

10 A. McCormick, P. Bombelli, R. Bradley, R. Thorne, T. Wenzel and C. Howe, Energy Environ. Sci., 2015, 8, 1092.

11 G. Uğur, J. Chang, S. Xiang, L. Lin and J. Lu, Adv. Mater., 2012, 24, 2685.

12 T. Park, J. Na, B. Kim, Y. Kim, H. Shin and E. Kim, ACS Nano, 2015, 9, 11830.

$13 \mathrm{X} . \mathrm{Yu}, \mathrm{J}$. Pan, J. Deng, J. Zhou, X. Sun and H. Peng, Adv. Mater., 2016, 28, 10744.
14 T. T. Zhao, W. T. Jiang, H. Z. Liu, D. Niu, X. Li, W. H. Liu, X. Li, B. D. Chen, Y. S. Shi, L. Yin and B. H. Lu, Nanoscale, 2016, 8, 8111.

15 X. Q. Wang, C. F. Tan, K. H. Chan, K. C. Xu, M. H. Hong, S. W. Kim and G. W. Ho, ACS Nano, 2017, 11, 10568.

16 J. J. Wie, D. H. Wang, V. P. Tondiglia, N. V. Tabiryan, R. O. Vergara-Toloza, L. S. Tan and T. J. White, Macromol. Rapid Commun., 2014, 35, 2050.

17 R. Tang, Z. Y. Liu, D. D. Xu, J. Liu, L. Yu and H. F. Yu, ACS Appl. Mater. Interfaces, 2015, 7, 8393.

18 W. Y. Wei, Z. W. Zhang, J. Wei, X. F. Li and J. B. Guo, Adv. Opt. Mater., 2018, 6, 1800131.

19 J. K. Mu, C. Y. Hou, B. J. Zhu, H. Z. Wang, Y. G. Li and Q. H. Zhang, Sci. Rep., 2015, 5, 9503.

20 L. D. Zhang, H. R. Liang, J. Jacob and P. Naumov, Nat. Commun., 2015, 6, 7429.

21 Y. B. Xiong, L. D. Zhang, P. Weis, P. Naumov and S. Wu, J. Mater. Chem. A, 2018, 6, 3361.

22 H. S. Nalwa, Ferroelectric Polymers: Chemistry, Physics, and Applications, Marcel Dekker, New York, 1995.

23 X. Chen, X. Han and Q. D. Shen, Adv. Electron. Mater., 2017, 3, 1600460.

24 D. Zabek, K. Seunarine, C. Spacie and C. Bowen, ACS Appl. Mater. Interfaces, 2017, 9, 9161.

25 H. Xue, Q. Yang, D. Y. Wang, W. J. Luo, W. Q. Wang, M. S. Lin, D. L. Liang and Q. M. Luo, Nano Energy, 2017, 38, 147.

26 Y. Zi, L. Lin, J. Wang, S. Wang, J. Chen, X. Fan, P.-K. Yang, F. Yi and Z. L. Wang, Adv. Mater., 2015, 27, 2340.

27 S. H. Wang, Z. L. Wang and Y. Yang, Adv. Mater., 2016, 28, 2881.

28 Y. Hu, Z. Li, T. Lan and W. Chen, Adv. Mater., 2016, 28, 10548.

29 C. Ohm, M. Brehmer and R. Zentel, Adv. Mater., 2010, 22, 3366.

30 T. J. White and D. J. Broer, Nat. Mater., 2015, 14, 1087.

31 H. K. Bisoyi and Q. Li, Chem. Rev., 2016, 116, 15089.

32 H. Zeng, O. M. Wan, P. Wasylczyk, R. Kaczmarek and A. Priimagi, Adv. Mater., 2017, 29, 1701814.

33 J. Lv, Y. Liu, J. Wei, E. Chen, L. Qin and Y. Yu, Nature, 2016, 537, 179.

34 A. H. Gelebart, D. J. Mulder, M. Varga, A. Konya, G. Vantomme, E. W. Meijer, R. L. B. Selinger and D. J. Broer, Nature, 2017, 546, 632.

35 M. Wang, B.-P. Lin and H. Yang, Nat. Commun., 2017, 8, 13981.

36 L. Q. Yang, K. Setyowati, A. Li, S. Q. Gong and J. Chen, Adv. Mater., 2008, 20, 2271.

37 R. R. Kohlmeyer and J. Chen, Angew. Chem., Int. Ed., 2013, 52, 9234.

38 Y. Yang, W. Zhan, R. Peng, C. He, X. Peng, D. Shi, T. Jing and Z. Lin, Adv. Mater., 2015, 27, 6535.

39 X. Liu, R. Wei, P. T. Hoang, X. Wang, T. Liu and P. Keller, Adv. Funct. Mater., 2015, 25, 3022.

40 Y. Yang, Z. Pei, Z. Li, Y. Wei and Y. Ji, J. Am. Chem. Soc., 2016, 138, 2118. 
41 X. L. Lu, H. Zhang, G. X. Fei, B. Yu, X. Tong, H. S. Xia and Y. Zhao, Adv. Mater., 2018, 30, 1706597.

42 W. Wang, X. M. Sun, W. Wu, H. S. Peng and Y. L. Yu, Angew. Chem., Int. Ed., 2012, 51, 4644.

43 X. M. Sun, W. Wang, L. B. Qiu, W. H. Guo, Y. L. Yu and H. S. Peng, Angew. Chem., Int. Ed., 2012, 51, 8520.

44 X. Xia, J. Chen, G. Liu, M. S. Javed, X. Wang and C. Hu, Carbon, 2017, 111, 569.
45 D. Niu, W. T. Jiang, H. Z. Liu, T. T. Zhao, B. Lei, Y. H. Li, L. Yin, Y. S. Shi, B. D. Chen and B. H. Lu, Sci. Rep., 2016, 6, 27366.

46 W. Y. Wei, A. S. Shi, T. H. Wu, J. Wei and J. B. Guo, Soft Matter, 2016, 12, 8534.

47 H. H. Xing, J. Li, Y. Shi, J. B. Guo and J. Wei, ACS Appl. Mater. Interfaces, 2016, 8, 9440.

48 C. Y. Wan and C. R. Bowen, J. Mater. Chem. A, 2017, 5, 3091. 49 D. J. Broer, J. Boven and G. N. Mol, Makromol. Chem., 1989, 190, 2255. 